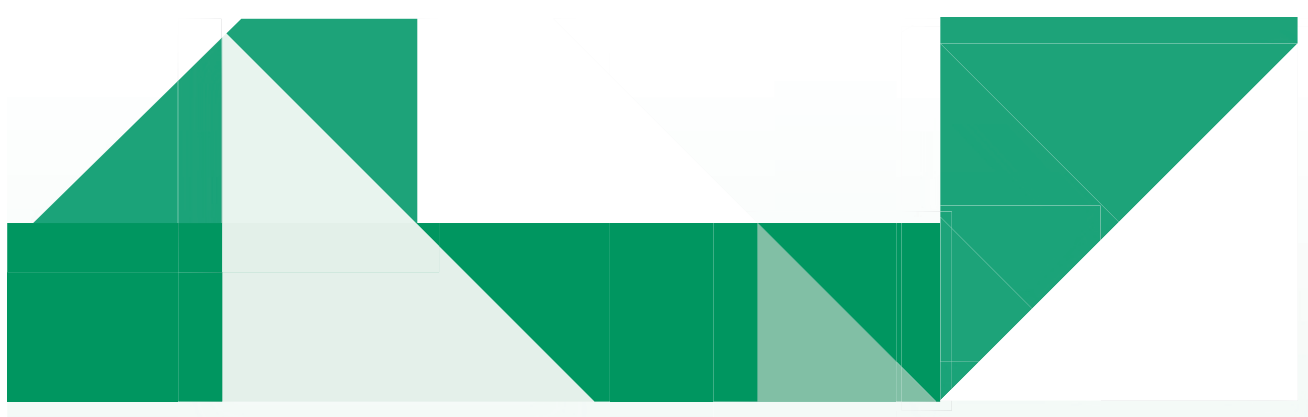

\title{
1) Estudio de caso en la enseñanza y aprendizaje de la fotosíntesis y respiración en plantas a partir de una unidad didáctica
}

\section{A Case Study in the Teaching and Learning of Photosynthesis and Respiration in Plants Based on a Teaching Unit}

Estudo de caso no ensino e aprendizagem da fotossíntese e respiração em plantas a partir de uma unidade de ensino

\section{Resumen}

La siguiente experiencia investigativa nace de la necesidad de resolver el problema ¿̇Cómo los estudiantes de séptimo grado de básica secundaria transforman conceptos científicos, procedimientos y actitudes hacia la ciencia en relación con la fotosíntesis y la respiración de las plantas a partir de su experiencia en actividades de aprendizaje de resolución de problemas y trabajos prácticos de laboratorio? Por lo tanto se presentan en este escrito las interpretaciones de la evolución y los cambios en los aprendizajes de una estudiante grado séptimo, en cuanto a los contenidos de ciencia escolar al desarrollar la unidad didáctica con actividades problémicas y experimentales, basada en la historia de la fotosíntesis y respiración en plantas que responde al objetivo general: desarrollar conceptos científicos escolares, predisposición positiva hacia la actividad científica y actitudes de aprendizaje hacia la biología y procedimientos propios de las ciencias, y visión sobre la naturaleza de la ciencia a través de la actividad científica escolar en el tema de fotosíntesis y respiración en plantas. El diseño metodológico, de carácter cualitativo, recurre al estudio de caso instrumental, que se fundamenta a través de antecedentes propios de la institución en los contenidos conceptuales y actitudinales. Además, fue necesaria la construcción, adopción y adaptación de instrumentos como entrevistas de ítem con escala pretest y postest, informes de laboratorio y una unidad didáctica para comprender la evolución del aprendizaje del estudiante en los contenidos como la concepción sobre la naturaleza de las ciencias. Es por esto que se recurrió a la triangulación en el tiempo, secuencia lineal-histórica, a partir de los estudios longitudinales y transversales para el análisis de la información, de tres casos seleccionados al azar, en esta nueva implementación. Este análisis sobre las actividades determinó que la evolución conceptual de los estudiantes pasa por explicaciones filogenéticas, y el cambio conceptual depende de la formu-
Héctor Alexánder Afanador Castañeda* Carlos Javier Mosquera Suárez ${ }^{* *}$

Docente catedrático de la Maestría en Didáctica de las Ciencias de la Universidad Autónoma de Colombia y docente de la Secretaría de Educación del Distrito. Correo electrónico: haacster@gmail.com. ORCID: orcid.org/0000-0001-9244-911X

** Docente del Doctorado en Educación de la Universidad Distrital Francisco José de Caldas. Correo electrónico: cmosquera@udistrital.edu.co.

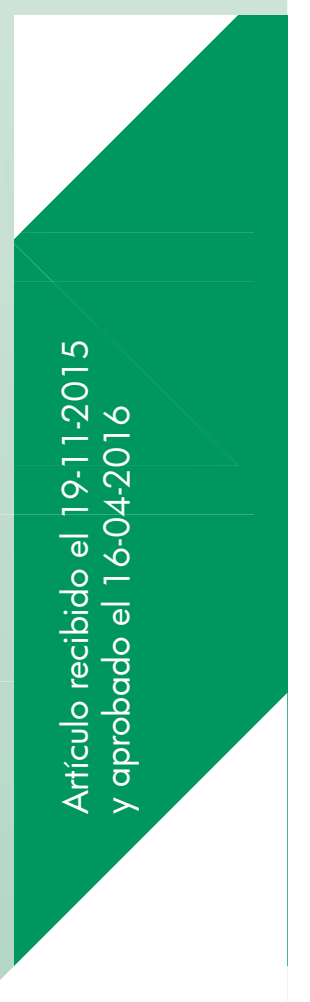


lación de explicaciones causales profundas. Además, se identificó la evolución procedimental a partir del nivel de abertura y cambio actitudinal hacia la ciencia influenciada por las actitudes de aprendizaje. Por último, los cambios y evoluciones en los contenidos favorecieron la tendencia de visión avanzada en la dimensión naturaleza del conocimiento científico.

Palabras clave

Contenidos de enseñanza y aprendizaje, historia de las ciencias, unidad didáctica

\section{Resumo}

A seguinte experiência de investigação decorre da necessidade de resolver o problema Como os alunos do sétimo da base secundária transformar conceitos científicos, procedimentos e atitudes em relação à ciência em relação à fotossíntese e respiração das plantas a partir de sua experiência em atividades aprendendo resolução de problemas e trabalhos de laboratório prático? Por isso, apresentada neste interpretações papel dos desenvolvimentos e mudanças na aprendizagem de uma sétima série do aluno, quanto ao conteúdo da ciência da escola para desenvolver a unidade de ensino com problémicas e actividades experimentais, com base no histórico de fotossíntese e respiração em plantas que satisfazem o objectivo geral: desenvolver conceitos de ciência da escola, predisposição positiva em relação à atividade científica e aprender atitudes em relação à biologia e procedimentos da ciência e insights sobre a natureza da ciência através da escola atividade científica na tema da fotossíntese e respiração das plantas. $\bigcirc$ desenho metodológico de natureza qualitativa, utiliza o estudo de caso instrumental, que é baseado através da sua própria história da instituição no conceitual e atitudinal também a construção, adoção e adaptação de instrumentos como item de entrevistas com escala preters e pós-teste, relatórios de laboratório e unidade didáctca foram necessários para compreender a evolução da aprendizagem dos alunos no conteúdo e concepção da natureza da ciência. É por isso que a triangulação foi usada no tempo, sequência linear-histórico, desde os estudos longitudinais e transversais para a análise de informação, três casos seleccionados aleatoriamente, esta nova aplicação. Esta análise das atividades determinou que o desenvolvimento conceitual dos alunos que passam por explicações filogenéticas e mudança conceitual depende da formulação de explicações causais profundas. Além disso, a evolução processual a partir do nível de abertura, e mudança de atitude em relação à ciência influenciada por atitudes identificadas aprendizagem. Finalmente, mudanças e desenvolvimentos no conteúdo favoreceu a tendência de visão avançada em dimensão natureza do conhecimento científico.

Palavras-chave

conteúdos atitudinais, conteúdo conceitual, conteúdo processual, história da ciência, unidade de ensino

\section{Abstract}

This research experience derives from the need to solve the problem "How do seventh-grade students transform scientific concepts, procedures and attitudes toward science in relation to photosynthesis and respiration of plants from their experience in problem-solving learning activities and practical laboratory work? This paper presents interpretations of the development and changes in learning of a seventh-grade student, as to the content of school science to develop the teaching unit with problem and experimental activities, based on the history of photosynthesis and respiration in plants, that meets the general objective: to develop school science concepts, positive predisposition towards scientific activity and learning attitudes towards biology and procedures of science, and insight into the nature of science through school scientific activity in the theme of photosynthesis and respiration in plants. The methodological design, of a qualitative nature, uses the instrumental case study, which is based through its own history of the institution in the conceptual and attitudinal contents. The construction, adoption and adaptation of instruments like item interviews with pretest and post-test scales, lab reports and a didactic unit were needed to understand the development of student learning in the content and conception of the nature of science. For this reason, we used 
triangulation over time, linear historical sequence, based on longitudinal and cross-sectional studies for the analysis of information of three cases chosen randomly in this new implementation. The analysis of the activities determined that the conceptual development of students passes through phylogenetic explanations, and the conceptual change depends on the formulation of deep causal explanations. In addition, we identified the procedural development from the opening level and the attitudinal change towards science influenced by learning attitudes. Finally, content changes and developments favored the trend of advanced vision in the nature dimension of scientific knowledge.

Keywords

Teaching and learning contents, history of science, teaching unit

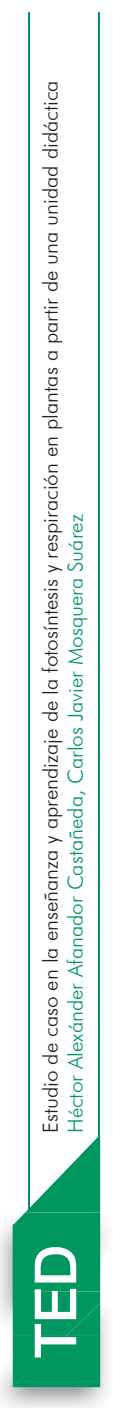




\section{Introducción}

La preocupación actual de la escuela gira en torno a la producción de conocimiento; se espera que las nuevas construcciones curriculares aporten a la alfabetización de la ciencia escolar (Maris, 2006 y Sanmartí, 2002). Entonces, al considerar el aprendizaje como un resultado de construcciones mentales basadas en conocimientos previos se cuestiona cómo los estudiantes de séptimo grado de básica secundaria transforman conceptos científicos, procedimientos y actitudes hacia la ciencia en relación con la fotosíntesis y la respiración de las plantas, a partir de su experiencia en actividades de aprendizaje de resolución de problemas y trabajos prácticos de laboratorio. La mayor dificultad para la enseñanza de las ciencias en la institución recae en la realización de actividades que contribuyan a un verdadero aprendizaje, de ahí que las nuevas prácticas deben incluir problemas interesantes y motivadores dentro del contexto de la historia de la ciencia, como facilitadores del aprendizaje de los contenidos (Afanador, 2011 y Pozo y Gómez, 1994).

La estrategia que se adopte en ciencias ha de adquirir la connotación de intencionalidad, es por esto que la implementación y ejecución de la unidad didáctica como experiencia de aula basada en resolución de problemas y trabajos prácticos sirve como herramienta didáctica para el desarrollo de capacidades de aprendizaje en los estudiantes de grado séptimo del Colegio Charry, ied, además como modelo alternativo de enseñanza de la biología (Afanador y Mosquera, 2013).

La misión de diseñar y aplicar una unidad didáctica que permita generar conocimiento escolar en los estudiantes de grado séptimo sobre el tema de la fotosíntesis y la respiración en las plantas busca relaciones entre el conocimiento previo y el nuevo conocimiento, además de la vivencia del aprendizaje de la ciencia escolar orientada en modelos didácticos por resolución de problemas y trabajos prácticos de laboratorio, el desarrollo de conceptos científicos de mayor nivel de profundización y poder explicativo, las habilidades para poner en práctica los conceptos adquiridos en situaciones cotidianas y la predisposición positiva hacia la actividad científica. Así, el propósito específico de esta investigación es identificar los cambios y evoluciones conceptuales a partir de las actividades problémicas de la unidad didáctica, determinar procedimientos, actitudes hacia la ciencia y la naturalezasobre la ciencia , cuando resuelven problemas cualitativos y problemas experimentales del estudio de caso perteneciente al grado séptimo.

\section{Marco de referencia}

El proceso de enseñanza/aprendizaje de la biología presenta ciertas dificultades en el desarrollo de ideas en profundidad, la conexión de ideas con la solución de problemas, la comprensión de procedimientos en trabajo práctico de laboratorio, la visión sobre la naturaleza de la ciencia, la amplitud de temas descontextualiza- 
dos y desarticulados (Maris, 2006), que recaen en uso discriminado del libro de texto como transposición de ciencia, que en muchos de los casos lleva a prácticas simplistas que repercuten en procesos de pensamiento estructural y complejo de la fotosíntesis y la respiración en las plantas (Gándara et al., 2002; González, García y Martínez, 2003) y concepciones erróneas de los estudiantes (Afanador, 2013; Charrier, Cañal y Rodrigo 2006; Çibik y Diken, 2008; Köse y Uşak, 2006). Por lo tanto, toda estrategia, incluidas las experiencias de aula, debe analizar y evaluar tres aspectos: lo que piensa el estudiante sobre lo que aprende; lo que hace, escribe y comunica; y el conocimiento escolar construido (Afanador, 2009).

Actualmente, se requieren modelos con tendencias contemporáneas que implementen el conocimiento didáctico del contenido a través de unidades didácticas (UD) como alternativa de solución a la problemática de aprendizaje. Desde Sánchez y Valcárcel (1993) y De Pro (1999), se interpreta que la UD es una alternativa a la enseñanza y aprendizaje pues es un sistema orgánico funcional y sinérgico entre los componentes que la conforman, cuyo propósito es dar respuesta a las necesidades escolares. A continuación, se ejemplifica la relación de los componentes de la UD a partir del contenido fotosíntesis y respiración en plantas: desde el componente de análisis científico, debe incluirse la historia de la ciencia para anticiparse a las dificultades conceptuales de los estudiantes con el ánimo de lograr una verdadera aprehensión de la génesis y evolución de los conceptos científicos (Wandersee, 1986). Para la enseñanza y aprendizaje, en especial de este contenido, se evidencia - desde una visión Toulminiana de génesis y evolución histórica conceptualla coexistencia de teorías. Según Toulmin (1977), el concepto depende de la "variación conceptual" y de la "selección" para obtener "variantes conceptuales", a lo que denominó "dinámica científica".

Entonces, la evolución conceptual depende de las variables ("representación transversal", "representación longitudinal" y al combinarlas dan la "representación evolutiva") que responden a los parámetros de innovación (explicaciones descriptivas) y normatividad (explicaciones causales). Desde el planteamiento evolucionista y para comprender el contenido, es necesario destacar las siguientes teorías: teoría del humus, teoría del intercambio gaseoso, teoría de la fotosíntesis como único proceso, teoría de nutrición de las plantas e independencia de la respiración como teoría de intercambio gaseoso, teoría de la combustión y la teoría de especiación que demuestra la interdependencia de los procesos a partir de las teorías de Hill, Calvin, Krebs y quimiosmótica. Además, es importante demostrar cómo estas han sufrido cambios conceptuales de forma gradual para la consolidación de teorías en los procesos de fotosíntesis y respiración.

Encontrada la anterior relación, se establece el componente de la selección de objetivos a partir de los intereses de los estudiantes y las intenciones del docente en los contenidos; por lo tanto se requiere identificar y determinar el cambio de concepto, la evolución de procedimientos para comprender el concepto, la predisposición hacia la ciencia y la relación de la naturaleza con la naturaleza de la ciencia dentro de la enseñanza y aprendizaje de la fotosíntesis y respiración en plantas.

Es fundamental que el componente estrategia didáctica responda a la construcción de conocimiento de ciencia escolar. Es por esto que el incorporar la resolución de problemas (RP) como actividad de investigación y proceso intelectual necesario para aprender ciencia en el aula debe dirigirse a la capacidad de enfrentar situaciones cotidianas con el propósito del 
cambio y evolución en los contenidos y comprensión sobre la naturaleza de la ciencia, involucrando las concepciones alternativas e históricas (Caballer, 1994; Pozo y Gómez, 1994). Esta estrategia debe incorporar los trabajos prácticos de laboratorio (TPL) en función del requerimiento de aprendizaje y como simuladores de la misma actividad científica (Gil y Valdés, 1996). Estos permiten que los objetivos sean inteligibles y mediadores entre el análisis científico y el análisis didáctico.

Por último, el componente de estrategias de evaluación busca la construcción y utilización de instrumentos para diagnosticar y evidenciar la evolución y la culminación del proceso de enseñanza y aprendizaje. El conjunto de actividades problemáticas y experimentales (UD) desde su orden lógico-histórico, y la secuencialidad y complejidad para desarrollar el aprendizaje del estudiante, según Sanmartí (2002), se convierten en los criterios para la evaluación formativa, siempre y cuando el estudiante distinga, comprenda y comparta lo aprendido con los demás; de lo contrario, el docente debe rediseñar las actividades. Además, la inclusión de instrumentos pretest y postest e instrumentos de seguimiento, permiten al estudiante valorarse a sí mismo, valorar al otro y ser valorado por otro, dado que toda actividad debe incluir los juicios de valor.

\section{Diseño metodológico}

En esta intervención investigativa cualitativa en enseñanza y aprendizaje se recurrió al diseño metodológico de estudio de casos, de tipo instrumental (véase la figura 1). Es importante señalar que en el contexto escolar, para interpretar la producción de datos, explicaciones y conductas observables en los contenidos y escenarios de aprendizaje como un todo de un contexto histórico y situacional (Stake, 1998), se requiere elaborar y rediseñar instrumentos para adaptarlos a las características de los estudiantes. Para esta investigación, las pruebas piloto y los diagnósticos se realizaron en octubre de 2010 y la ejecución o aplicación durante los tres periodos académicos del 2011. Cabe decir que el diagnóstico de Afanador (2009) y los hallazgos previos a esta investigación en actitudes hacia la ciencia y actitudes de aprendizaje y concepciones alternativas sobre la fotosíntesis y la respiración publicados por Afanador y Mosquera (2012 y 2013, respectivamente), justificaron el diseño metodológico y la aplicación de la unidad didáctica (véase http://es.slideshare.net/DidacticaCienciasUAC/unidad-didctica-13439242) y demás instrumentos, como alternativa de solución a nuestro problema de investigación.

Los instrumentos empleados requirieron del soporte o fundamento teórico, para el cual se seleccionaron investigadores como Sánchez y Valcárcel (1993), De Pro (1999) y Sanmartí (2002) para el diseño de la UD. Además, se incluyó la visión Toulminina para construir la red sistémica y demostrar la coexistencia de explicaciones científicas en la historia de la ciencia sobre el concepto de fotosíntesis y respiración en plantas. Según Toulmin (1977), en la "dinámica 
científica", el cambio conceptual depende de las variables "representación transversal" y "representación longitudinal", que al combinarlas dan cuenta de la "representación evolutiva" o explicaciones normativas o causales. En la figura 2 se representa la evolución conceptual; allí se evidencia la coexistencia de teorías y la aparición de "buenas razones" o "casos nebulosos" que responden a las diferentes clases de explicaciones y cómo estas han sufrido cambio conceptual de forma gradual para la consolidación de teorías. El propósito de la UD fue relacionar el momento histórico-teórico del estudiante (concepciones alternativas) y el desarrollo filogenético por el cual debe transitar (secuencia de actividades) para comprender el concepto y avanzar en el aprendizaje.



Figura 1. Diagrama de las fases del proceso investigativo

Para el caso de los otros instrumentos, como el informe de laboratorio, se tuvo en cuenta a Chamizo (2009). Mientras las entrevistas de escala fueron adoptadas y adaptadas de las investigaciones de Adúriz-Bravo (2001), Leymonié (2009), Charrier, Cañal y Rodrigo (2006), Vázquez y Manassero (1997), Manassero y Vázquez (2001), Gargallo et al. (2007), Pozo y Gómez (1994) y Del Carmen (2006), con el propósito de analizarlos a partir de comparaciones de cambio en los ítems. Se recurrió a la triangulación en el tiempo (Cohen y Manion, 1990) de tipo secuencia lineal-histórica para la síntesis de los casos de estudio.

La investigación y aplicación de los instrumentos se realizó en el colegio Charry IED de la localidad décima de la ciudad Bogotá, donde se intervino el grado séptimo de básica secundaria. Participaron 26 mujeres y 18 hombres, cuyas edades oscilan entre los 12 y los 15 años, de los cuales se seleccionaron tres casos al azar. 


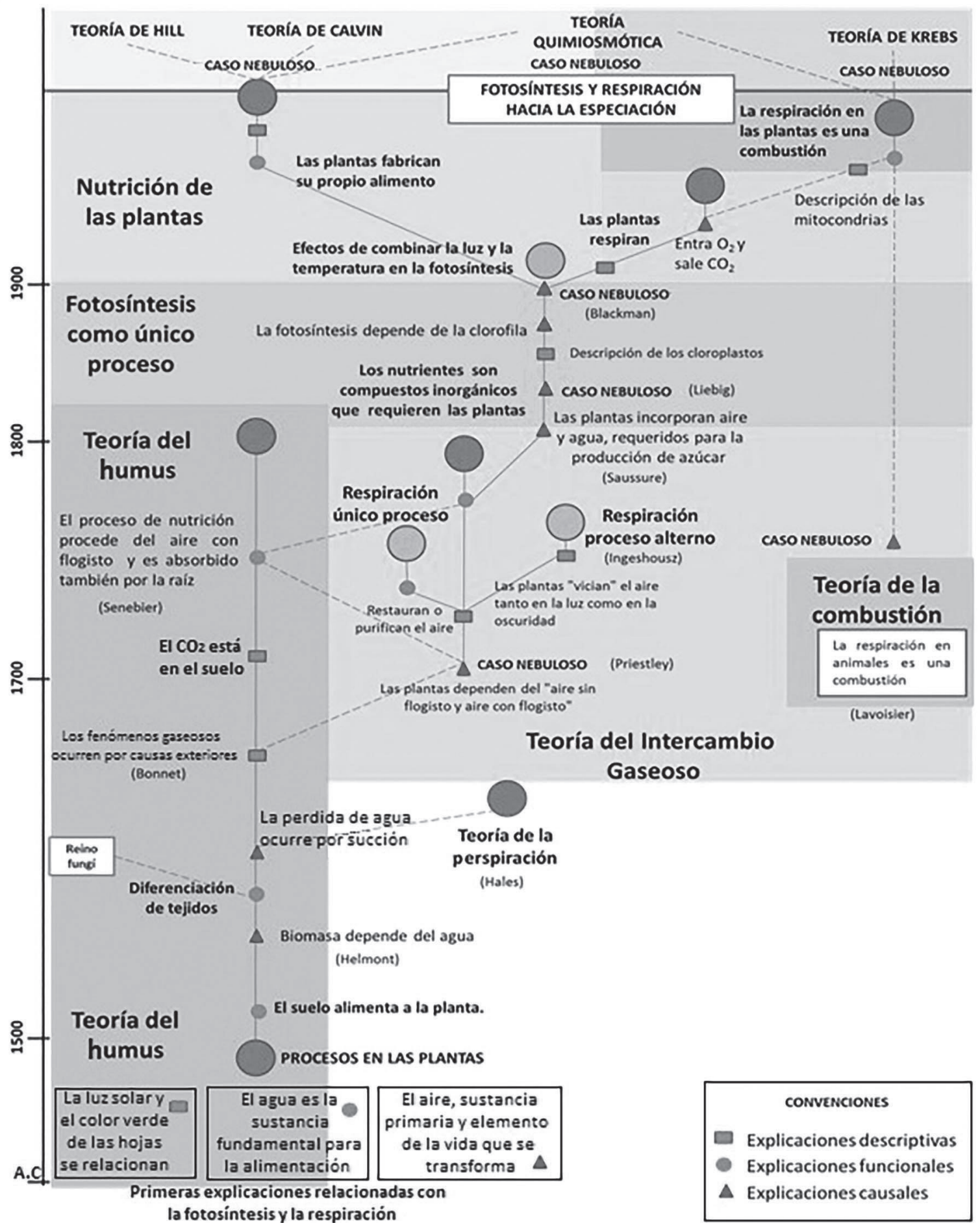

Figura 2. Evolución de las explicaciones científicas sobre fotosíntesis y respiración en plantas desde una visión toulminiana 


\section{Resultados y discusiones}

En esta sección del documento se describen solo los datos del caso Laura y sus interpretaciones en el contenido actitudinal, contenido procedimental, naturaleza de la ciencia y el contenido conceptual como evidencia de la investigación, luego se presenta la síntesis de este caso.

En el contenido actitudinal hacia la ciencia, se identificó (tanto en Laura como en los otros dos casos) una tendencia favorable hacia las categorías imagen de ciencia y medioambiente en el pretest y en el postest. Los estudiantes consideraron que la ciencia es importante para la sociedad, para el desarrollo de un país, y se traduce en mejores oportunidades para las generaciones futuras. También opinan que la ciencia puede resolver los problemas del medioambiente y ven la necesidad de aprender sobre ella para aumentar el aprecio por la naturaleza. En la figura 3 se describen las categorías ciencia escolar y disciplina de estudio, del caso Laura.



Figura 3. Tendencia comparativa en actitudes hacia la ciencia de las categorías ciencia escolar y disciplina de estudio.

En la figura 3 se compara la dispersión de los indicadores de actitudes hacia la ciencia, antes y después de la intervención con la unidad didáctica. De acuerdo con los indicadores rojos (después de la intervención), Laura presenta cambios en ciertos indicadores que afectan en especial la categoría de actitudes disciplina de estudio. Es decir que la unidad didáctica (conjunto de actividades) que tiene una intencionalidad en el proceso de aprendizaje modifico la pasión por la ciencia, en especial la biología, o el interés por estudiar y laborar en el campo de las ciencias biológicas. Este cambio es por causa de los indicadores it 13 ("la ciencia escolar me ha abierto los ojos a nuevos y excitantes mundos") e it22 ("la ciencia escolar me ha demostrado la importancia de la ciencia para nuestra manera de vivir") que cambiaron a una tendencia indecisa.
Al afirmar, en el caso Laura, que hubo cambios en ciertos indicadores, y presenta una predisposición positiva hacia la ciencia, es importante considerar que las actitudes hacia la ciencia dependen de las actitudes de aprendizaje. Por lo tanto es importante identificar las actitudes de aprendizaje hacia la Biología.

En el caso Laura, en la figura 4 se presenta la dispersión de tendencia después de la intervención con la unidad didáctica. De acuerdo con los indicadores actitudinales expuestos, están divididos en los siguientes grupos: tendencia aprendida positiva (muy favorable y favorable) y tendencia aprendida con dificultad (indecisa). 



Figura 4. Registro de tendencia de las actitudes de aprendizaje hacia la Biología Postest.

Como se ve en la figura 4, el indicador it6 ("desarrollo cosas originales en las actividades de aprendizaje de biología") con tendencia aprendida con dificultad, se ve afectado debido a que las habilidades en la resolución de problemas son adquiridas de forma inmediata, como lo demuestran sus indicadores actitudinales (it12, "al resolver un problema hago un plan", e it14, "me gusta trabajar en un problema difícil de Biología"). Esto afecta la motivación de la estudiante repercutiendo en el esfuerzo personal (it19, "encuentro nuevas cosas en Biología para estudiar sin ayuda", e it20 "estudio situaciones desconocidas para tratar de entenderlas"). Y en el trabajo en equipo, como lo demuestran los indicadores it27 e it29 ("mi trabajo en el aula es constante y enriquece al grupo o equipo de trabajo" y "tengo buena disposición para la construcción y presentación de informes de laboratorio", respectivamente).

Al relacionar los indicadores con tendencia de aprendizaje con dificultad (it12, it19, it20, it27 e it29) de las actitudes de aprendizaje hacia la biología se puede establecer que en el caso Laura incidieron notablemente en los indicadores con tendencia indecisa (it13 e it22) de las actitudes hacia la ciencia, además del cambio de la tendencia muy favorable a favorable en las actitudes en la categoría disciplina de estudio.

Es importante agregar dentro de estos resultados actitudinales los juicios de valor de los casos de estudio, puesto que estos ratifican los cambios o progresos que han logrado los estudiantes participantes en su proceso de aprendizaje al desarrollar las actividades de la UD. En consecuencia, para dar una mayor interpretación a estos juicios de valor se agruparon en núcleos de actividades.

En la figura 5 se indica en el primer núcleo (actividades problémicas sobre la historia de la ciencia) una motivación intrínseca positiva de Laura en las actividades. Los juicios de valor expresados resaltan el contenido actitudinal de aprendizaje hacia la biología. Esta motivación intrínseca de aprendizaje dependió de la atracción con que fueron construidas las actividades históricas sobre la fotosíntesis y la respiración de las plantas ("me gustó lo que investigaron, lo que hicieron [los científicos] fue muy divertido"), lo que permitió un progreso en el contenido procedimental (fue fácil encontrar la información) y la evolución conceptual ("cambié mis concepciones"). 


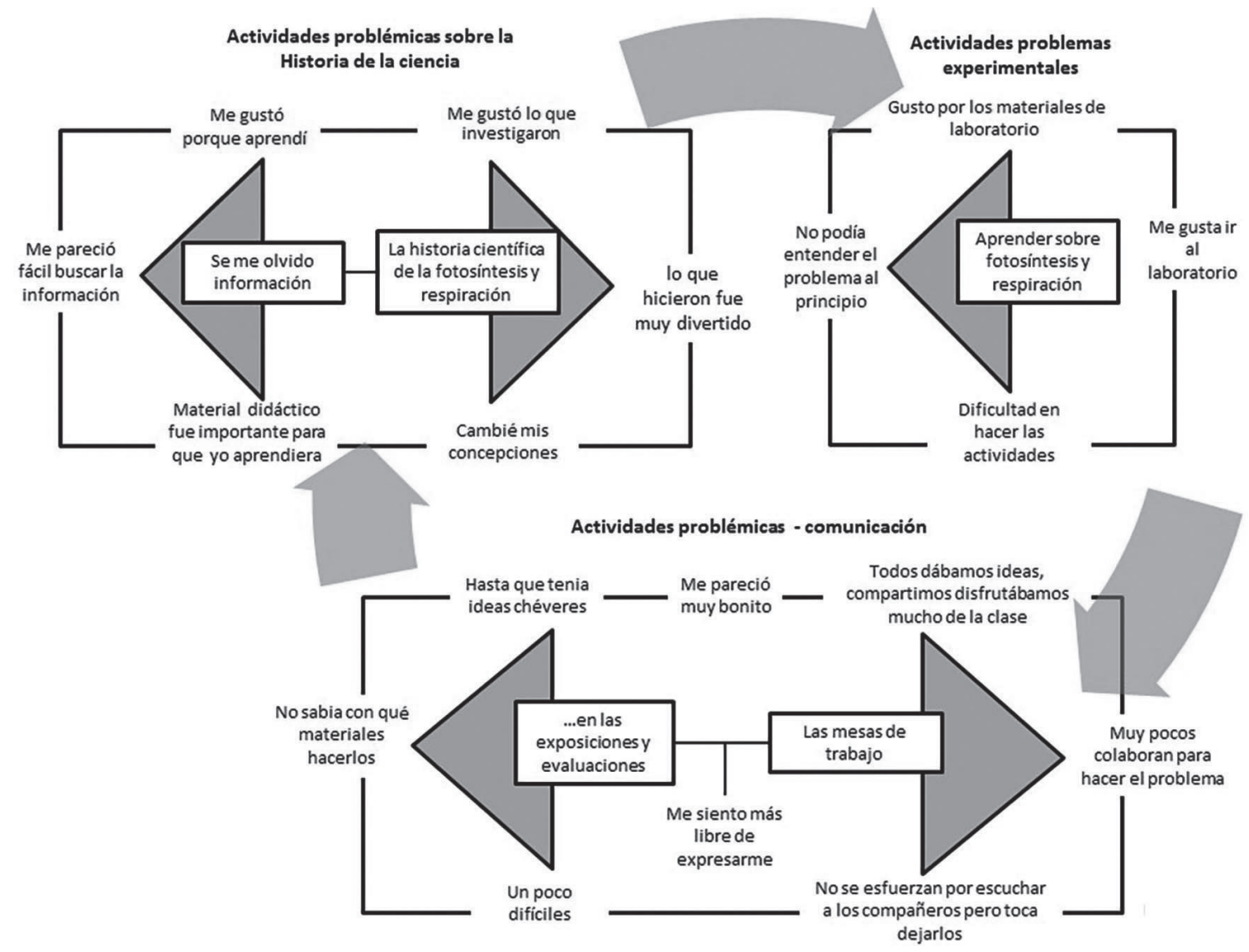

Figura 5. Juicios de valor de la estudiante Laura frente al desarrollo de las actividades de la UD.

En el segundo núcleo (problemas experimentales), la motivación intrínseca disminuyó puesto que sus actitudes de aprendizaje, en especial las de resolución de problemas, fueron afectadas por el contenido procedimental, como lo demuestran sus juicios de valor ("no podía entender el problema al principio" y "dificultad en hacer las actividades").

En el núcleo actividades problémicas se identifica una evolución positiva en la motivación intrínseca puesto que esta no fue afectada por factores externos (la actitud de los compañeros ante las actividades); por el contrario, adquirió aprendizajes de ciencia escolar como la tolerancia, la participación y la comunicación. Esta evolución inició con la dificultad del contenido procedimental ("actividades un poco difíciles" y "no sabía con qué materiales hacerlos") hasta llegar a lograr el éxito en la actividad ("me siento más libre de expresarme"), como lo demuestra su actitud de aprendizaje ("me pareció muy bonito").

De acuerdo con los juicios de valor de Laura, se establece que la motivación intrínseca es esencial en la evolución de los aprendizajes, de tal modo que si la esta disminuye, se afectarán las tendencias en las actitudes de aprendizaje $y$, por consiguiente, las actitudes hacia la ciencia, en especial las de disciplina de estudio. 


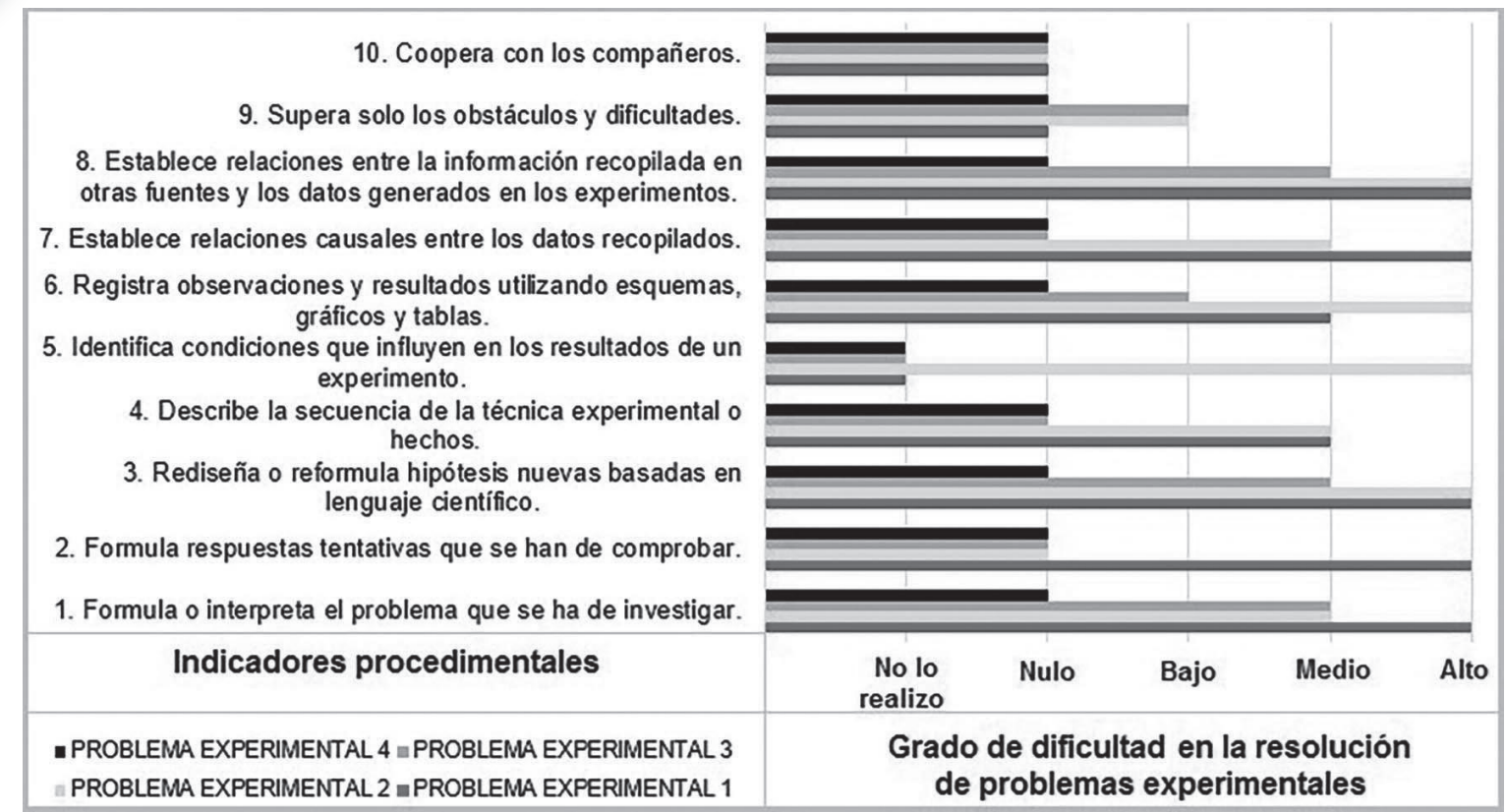

Figura 6. Historial de procedimientos y su nivel de dificultad del caso Laura

En el contenido procedimental del estudio de caso (véase la figura 6), se evidencia un proceso progresivo en su aprendizaje y en la adquisición de los indicadores procedimentales. Teniendo en cuenta el desempeño en los indicadores, la estudiante se clasificó en el nivel de abertura de Herron (1971) y Priestley (1992).Al respecto, en Herron (1971), toda actividad que contenga carga experimental debe plantear e identificar un problema que debe ser solucionado por medio del diseño y desarrollo de la estrategia experimental con la finalidad de obtener una respuesta. En Priestley (1997), se basa en la proporción en la que el docente facilita los problemas, las maneras y medios para afrontar el problema y la respuesta a esos problemas. Por lo tanto, a mayor participación del docente menor será el nivel de abertura del estudiante (citados en Jiménez, Llobera y Llitjós 2006).

En el problema experimental uno se establece que la estudiante estuvo en el nivel de abertura de Herron cero (requirió intervención del docente para resolver el problema con un método establecido) y desde el nivel de abertura de Priestley correspondió al tipo cerrado (se le proporcionaron todos los procedimientos). Para el problema experimental dos, el nivel de abertura de Herron fue uno (requirió de instrucciones para el dominio de técnicas experimentales) y en el nivel de abertura de Priestley semi-cerrado (se le proporcionaron algunos procedimientos). En el caso del problema experimental tres el nivel de abertura de Herron fue el nivel dos (planificó el experimento e identificó variables, para representarlas dentro de una gráfica cualitativa), pero desde el nivel de abertura de Priestley respondió al nivel abierto (desarrolló sus propios procedimientos, se le proporcionó el método, y realizó los procedimientos para elaborar las generalidades). Este último resultado también se hace evidente en el cuarto problema experimental. 
En las tendencias de los problemas experimentales se observa que la estudiante no tenía experiencia en los procedimientos que se requieren en la resolución de problemas experimentales, pero tenía gran predisposición al trabajo en equipo (it 10). Al comparar los problemas experimentales, es notorio que los dos primeros presentaron una similitud en el nivel de abertura, de tal modo que requirió el acompañamiento permanente del docente. Es importante resaltar que la estudiante abandona el indicador "identificación de condiciones que influyen en los resultados de un experimento" luego de no tener evoluciones de aprendizaje en él; pero el abandonar este indicador no indica un obstáculo para la adquisición en los otros indicadores procedimentales ni en el aprendizaje del contenido procedimental.

Al relacionar los progresos o evoluciones en motivación intrínseca, actitudes de aprendizaje hacia la biología y actitudes hacia la ciencia, se puede establecer claramente que el contenido procedimental influye sobre la dinámica entre estos tres componentes del contenido actitudinal, como lo demuestran sus juicios de valor. Es decir, el aprendizaje evolutivo en el contenido procedimental de Laura, que inició de mayor a menor grado de dificultad en los indicadores procedimentales, donde involucramos el grado de abertura, hizo que se presentaran cambios (disminución y aumento) en la motivación intrínseca, los cuales repercutieron en algunas actitudes de aprendizaje hacia la biología que fueran difíciles de aprender, además afectaron el cambio de Muy favorable a Favorable en las tendencias en las actitudes hacia la ciencia.

Con respecto a la visión sobre la naturaleza de la ciencia, en el caso Laura se identifican tendencias diferentes en los criterios en cada una de las categorías: relación teorías y hechos, naturaleza del conocimiento científico y desarrollo del conocimiento científico (véase la figura 7).

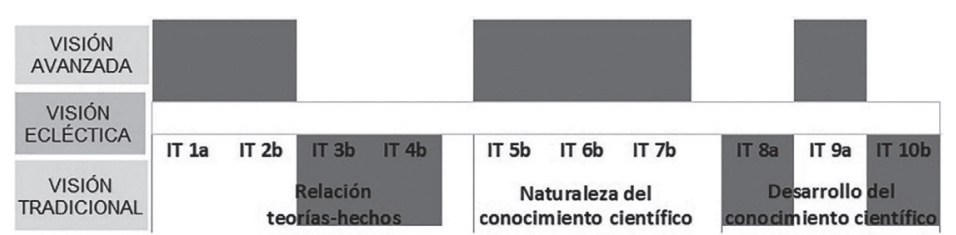

"la ciencia se hace en un laboratorio, esta sirve para dar una mejor respuesta a un problema, y la ciencia se hacen conclusiones o respuestas, y se juntan para hacer una mejor". Entonces los científicos saben mucho porque sea el problema que sea le buscan solución".

Figura 7. Tendencia postest sobre la naturaleza de la ciencia y opinión sobre qué es la ciencia.

La estudiante Laura en la categoría "relación de teoría y hechos" presentó dos visiones: tradicional y avanzada. Desde la visión tradicional tiende a considerar que una teoría es una hipótesis que se ha confirmado y el progreso científico consiste en descubrir teorías que se aproximen a la verdad ("dar una mejor respuesta a un problema"). Pero desde la visión de avanzada, piensa que las teorías son invenciones de los científicos ("la ciencia se hacen conclusiones o respuestas"); en esta medida, las afirmaciones de los científicos están influenciadas por la comunidad científica y por investigaciones anteriores (según los juicios de valor, en el trabajo en equipo "todos damos ideas").

En la dimensión "naturaleza del conocimiento científico", la estudiante tendió a reconocer que existen diferentes metodologías científicas, que se adoptan de acuerdo a las circunstancias. En estas metodologías las leyes se validan por 
consenso de los científicos (como argumenta a través de los juicios de valor "se hace en el laboratorio..." y "se juntan para hacer una mejor conclusión o respuesta"; esta concepción es propia del trabajo en equipo y responde a una visión de avanzada).

En la dimensión "desarrollo del conocimiento científico", la concepción de la estudiante tiende a considerar que la ciencia ha evolucionado mediante la acumulación de teorías verdaderas y el progreso científico consiste en descubrir teorías que se aproximen cada vez más a la verdad (se evidencia esta tendencia de visión tradicional en el argumento de la estudiante "sea el problema que sea le buscan solución"). Paralelamente consideró desde una visión de avanzada que el conocimiento científico es tentativo.

El desarrollo de las actividades de la UD mostró que la estudiante obtuvo dos visiones sobre la naturaleza de las ciencias, pero su opinión sobre la ciencia también demuestra una visión pragmática-utilitaria. Esta visión fue adquirida a través del progreso en el contenido procedimental (del mínimo grado de abertura al máximo), hipotéticamente puede decirse que si realizara otra cantidad de actividades de problemas experimentales aumentaría su visión de avanzada sobre la naturaleza de la ciencia, puesto que pasó de un modelo inductivo a un modelo deductivo de forma progresiva. Además, su opinión desde la visión pragmática sobre la ciencia ("los científicos saben mucho porque sea el problema que sea le buscan solución") ratifica la tendencia favorable hacia la ciencia, en especial la categoría ciencia escolar.

Se estableció que los estudiantes participantes, antes de la intervención, no tenían una verdadera comprensión de la fotosíntesis y respiración en las plantas. En la figura 8se interpretan los criterios de Laura.

En la primera gráfica de dispersión (figura 8a), en el criterio uno, Laura presentó una tendencia desfavorable hacia la comprensión en el reconocimiento de estructuras celulares y químicas que participan en el proceso de la fotosíntesis y la respiración. Las concepciones alternativas se centran en la diferenciación de las funciones de los organelos celulares de la fotosíntesis y la respiración y en atribuirle a las hojas la función de respiración y fotosíntesis. Frente al criterio dos, expresó un bajo reconocimiento de conceptos y características propias de las plantas asociados a la fotosíntesis. Además, mostró una tendencia favorable de comprensión para reconocer como característica que las plantas fabrican su propio alimento, pero desligó esta explicación de los conceptos autótrofo, fotosíntesis y productor. En el criterio tres existió una baja tendencia de comprensión para identificar la influencia de la luz en el proceso de la fotosíntesis, además desconoció aspectos específicos de la incidencia de la luz en el proceso de la fotoquímica; evidencia de ello es la concepción frente a la producción del oxígeno ya que la confundió con respiración. 
Tendencia de concepciones del pretest sobre fotosíntesis y respiración en planta.

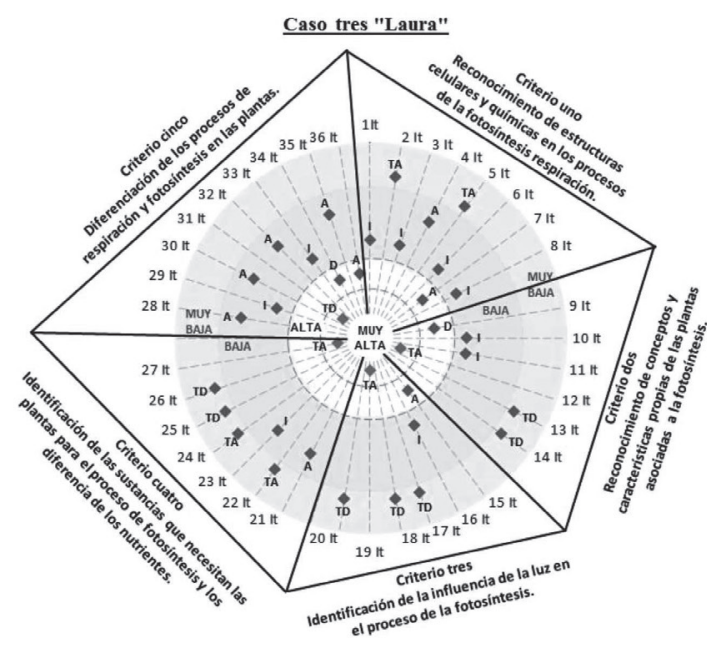

Tendencia de concepciones del postest sobre fotosíntesis y respiración en planta.

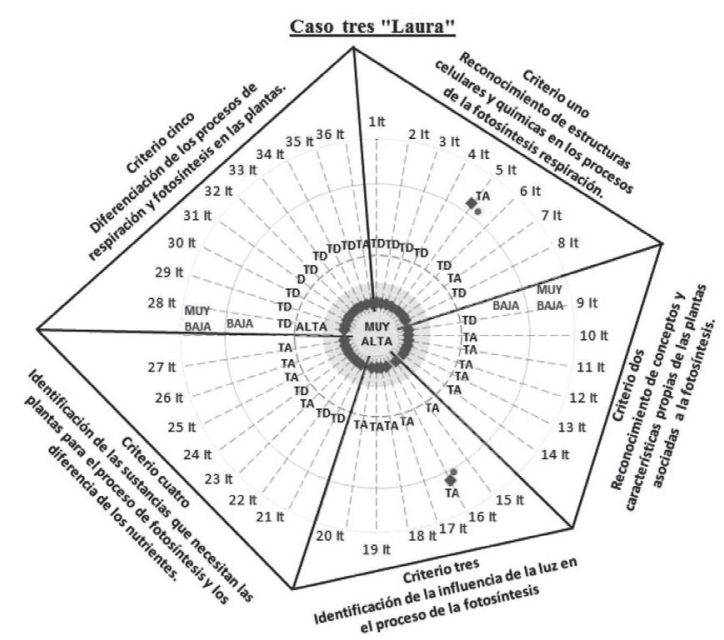

Figura 8. Tendencia comparativa de concepciones sobre fotosíntesis y respiración en plantas del caso tres "Laura".

En las figuras $8 a$ y $8 b$ se representa la relación de migración de los indicadores en cada criterio (en la figura 8 a se describe la baja tendencia de comprensión y en la figura $8 \mathrm{~b}$ la alta tendencia comprensión del contenido); allí se establece tendencia evolutiva conceptual en los criterios y se demuestra el cambio conceptual sobre el contenido. Se aclara que la teoría quimiosmótica no se comprende en su totalidad, debido a la muy baja comprensión de la relación entre clorofila y onda espectral visible (it5 e it 16), que para grado séptimo es un contenido de alto grado de complejidad que requiere de mayor tiempo y nuevas secuencias de actividades.

En el criterio cuatro, Laura tuvo tendencia desfavorable hacia la comprensión en identificación de las verdaderas sustancias que necesita la planta para el proceso de fotosíntesis, al considerar que las plantas requieren alimentarse del suelo (nutrientes procesados o fertilizantes). Esta concepción hace ver la confusión entre alimentos y nutrientes (afirma que los alimentos para ella son fertilizantes, agua y sol), por ende no relaciona fotosíntesis con nutrición. Y en el criterio cinco es notoria la baja tendencia de comprensión para la diferenciación entre la fotosíntesis y la respiración puesto que no identifica la función y el proceso con el concepto (el crecimiento de la planta, la importancia de la glucosa, las plantas no respiran, las plantas respiran de noche, etc.).

Las explicaciones de la estudiante demuestran que hubo un avance en las explicaciones científicas o evolución histórica desde la visión toulminiana. Su evolución conceptual parte de incorporar nuevos conceptos específicos que le permitieron identificar características propias de las plantas y abandonar la teoría del humus. Su interpretación causal del modelo explicativo de especiación fue fundamental como principio de progreso conceptual y complementario al modelo experimental para la comprensión de variables (Laura filogenéticamente pasó a la teoría fotosíntesis como único proceso). Esta estabilidad cambió cuando formuló la teoría de gases como explicación causal del proceso de respiración; entonces, la evolución conceptual se situó en teorías independientes. La anterior teoría se 
desestabilizó cuando la estudiante (Laura) formuló una explicación funcional a partir de relacionar variables (glucosa - ATP, $\mathrm{H}_{2}$ y $\mathrm{O}_{2}-\mathrm{H}_{2} \mathrm{O}$, glucosa $-\mathrm{CO}_{2}$ ) en la solución de problemas. En un comienzo relacionó $\mathrm{CO}_{2}$ y $\mathrm{O}_{2}$ como una explicación cotidiana, lo que le permitió reelaborar la explicación causal para diferenciar el proceso y construir la generalidad de interdependencia en función de la respiración, "la glucosa sale del cloroplasto, en el citoplasma libera dos ATP. Se divide en dos moléculas que se van a la mitocondria. En el ciclo de krebs se obtiene $\mathrm{CO}_{2}$ y ATP, se necesita agua...". La evolución conceptual de Laura terminó en un cambio conceptual (figura 8b), gracias a que diferenció el proceso de intercambio gaseoso de fotosíntesis y de respiración demostrada en la generalización final para la apropiación de los conceptos en una situación real y teórica (véase la figura 9).
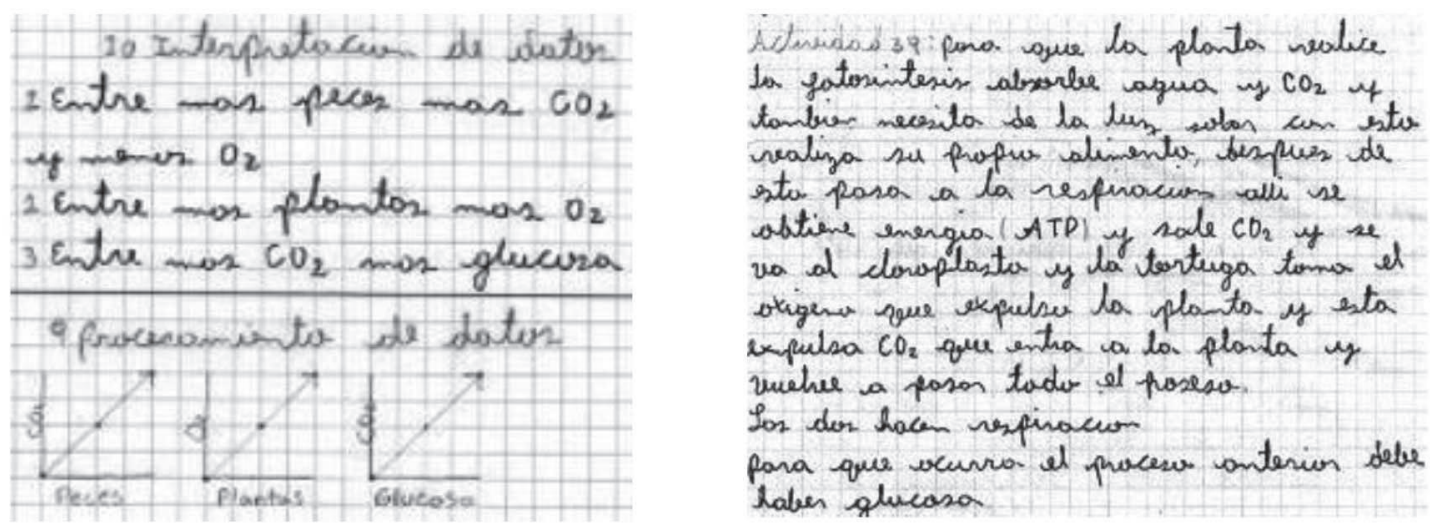

Figura 9. Procesamiento e interpretación de datos en la actividad de construcción del acuario y actividad 39 de generalización

\section{Síntesis del aprendizaje de los contenidos: caso Laura}

Este cambio conceptual dependió de la evolución del aprendizaje procedimental. Laura demostró habilidad para buscar y seleccionar información y confrontar sus concepciones con el conocimiento científico y, en consecuencia, evolucionó en el nivel de abertura. Con relación a los niveles de abertura, la estudiante inició en el nivel uno -cerrado (requirió intervención del docente para resolver el problema y se le proporcionaron los procedimientos)- y avanzó de forma gradual hasta el nivel tres -muy abierto (solo se le proporcionó el problema)-; por lo tanto, presentó cambio procedimental en el que se establece que adquirió un método deductivo.

Laura, al desarrollar las actividades problémicas, adquirió actitudes de aprendizaje hacia la biología, tales como como regulación, responsabilidad, creatividad, desarrollo cognitivo, iniciativa, comunicación y desarrollo social, los cuales permitieron estabilidad y favoritismo en las actitudes hacia la ciencia, en especial las categorías de ciencia escolar, lo que contribuyó a su vez en la toma de decisiones, una mayor apertura cognitiva, control sobre el propio aprendi- 
zaje, utilidad para seguir posteriores estudios y disponibilidad e interés hacia la ciencia, lo que redunda en la motivación hacia su estudio. Pero el aprendizaje de este contenido, incluidos los otros, se logró debido a la motivación intrínseca de la estudiante, gracias a la que factores como el compromiso, la participación y el diálogo fueron esenciales.

El desarrollo de las actividades problémicas y los cambios positivos en los contenidos y la evolución de los aprendizajes permiten establecer que en Laura coexisten la visión tradicional (con mayor tendencia) y la visión avanzada sobre la naturaleza de la ciencia, pues tendió por el realismo ingenuo, la influencia de la comunidad científica y el acumulacionismo como modelo de cambio científico

\section{Conclusiones}

La estrategia de enseñanza y aprendizaje permitió identificar las concepciones, el nivel de profundidad y el poder explicativo de las construcciones sobre el contenido (de acuerdo con la comprensión de los estudiantes del estudio de caso), la movilización de procedimientos aprendidos y las reflexiones sobre las actitudes, a partir de los procesos de interacción con las actividades. Además, contribuyó a la visión de avanzada de la estudiante Laura sobre la naturaleza de la ciencia en las categorías Relación de teorías y hechos y Naturaleza del conocimiento científico, en las que se determinó que la ciencia responde a un sistema conceptual y los hechos construidos están cargados de teoría.

A partir del estudio del caso Laura, al igual que en los otros dos casos no mencionados aquí, se establece que la comprensión de un concepto depende de la apropiación del mismo para aplicarlo en otro contexto, por lo tanto el cambio conceptual está determinado por el grado de estabilidad de sus concepciones y los factores socioculturales, puesto que las creencias con las que llegan al aula de ciencias obstaculizan la comprensión sobre la relación entre fotosíntesis y respiración, o contribuyen a la coexistencia de dos concepciones: cultural y científica.

El lenguaje científico permite incorporar nuevos conceptos a las explicaciones de los estudiantes, siempre y cuando estos sean comprensibles, traducidos en explicaciones descriptivas. Solo cuando han adquirido todos sus atributos de forma secuencial, se expresan en explicaciones funcionales o explicaciones causales como acto reflejo de aprendizaje. De no darse esta situación, la estudiante vuelve a la explicación teleológica y cotidiana del intercambio gaseoso, gracias a sus creencias y a la influencia cultural.

El contenido procedimental del estudio de caso Laura respondió a un aprendizaje progresivo, en el cual su estrategia para la solución de actividades problémicas se fue consolidando a medida que iba realizando más actividades de este tipo por sí sola. Al igual que en los otros casos de estudio, es importante precisar que los estudiantes llegan con baja experiencia en este tipo de estrategia; en consecuencia presentan menor nivel de abertura y, por ende, dependencia del profesor. Se establece entonces que el aprendizaje de los procedimientos está sujeto a la dinámica y el protagonismo de sus actores y al nivel de dificultad del problema, y la evolución del aprendizaje procedimental está determinada por el nivel de dificultad del problema experimental.

La estrategia de enseñanza y aprendizaje diseñada promueve actitudes positivas de aprendizaje hacia la biología y hacia la ciencia. Se identificó que el grado de estabilidad de las actitudes de aprendizaje hacia la biología como responsabilidad, regulación y 
creatividad (aprendizaje hacia la autonomía), actitudes de desarrollo cognitivo, estrategia y motivación (aprendizaje hacia la resolución de problemas), actitudes de esfuerzo personal, iniciativa y comunicación (aprendizaje hacia las actividades) y actitudes de cooperación y desarrollo social (aprendizaje hacia el trabajo en equipo) son determinantes para que haya una verdadera predisposición hacia la ciencia con respecto a ciencia escolar y disciplina de estudio. Por lo tanto, el verdadero aprendizaje requiere de actitud positiva, interés y voluntad, elementos constitutivos de la motivación intrínseca.

\section{Referencias bibliográficas}

Adúriz-Bravo, A. (2001). Integración de la epistemología en la formación del profesorado de ciencias (tesis de doctorado). Universidad Autónoma de Barcelona, España. Recuperado de http://tdx.cat/handle/10803/4695.

Afanador, H. (2009). Experiencia de aula "huerto escolar", estrategia para desarrollar el conocimiento escolar. Ponencia en el I Congreso Nacional de Investigación en Educación en Ciencias y Tecnología. Bogotá: Asociación Colombiana para la Investigación en Ciencias y Tecnología Educyt.

Afanador, H. (201 1). La enseñanza y aprendizaje de la fotosíntesis y respiración en plantas a partir de resolución de problemas y trabajos prácticos de laboratorio, unidad didáctica. Publicado en recurso educativo abierto Didáctica Ciencias UAC de la comunidad Slideshare. Recuperado de http://es.slideshare.net/DidacticaCienciasUAC/unidad-didctica-13439242.

Afanador, H. y Mosquera, C. (2012). Valoración de actitudes hacia la ciencia y actitudes hacia el aprendizaje de la biología en educación secundaria. Revista Bio-grafía (DBI), 5(8), 32-49.

Afanador, H. y Mosquera, C. (2013). Diagnóstico de concepciones alternativas sobre fotosíntesis y respiración en plantas. Revista de Educación, Pedagogía y Ciencias de la Universidad Autónoma de Colombia, 2(1), 89-105.

Caballer, M. (1994). Resolución de problemas y aprendizaje de la geología. Enseñanza de las Ciencias de la Tierra, 2(2), 393-397.

Chamizo, J. (2009). Historia Experimental de la Química. Tecné, Episteme y Didaxis. Iv Congreso Internacional sobre Formación de Profesores de Ciencias, número extraordinario, 7-16.

Charrier, M.; Cañal, P. y Rodrigo, V. (2006). Las concepciones de los estudiantes sobre la fotosíntesis y la respiración: una revisión sobre la investigación didáctica en el campo de la enseñanza y el aprendizaje de la nutrición de las plantas. Enseñanza de las Ciencias, 24(3), 401-410. 
Çibik, A. y Diken, E. (2008). The effect of group works and demonstrative experiments based on conceptual change approach: Photosynthesis and respiration. Asia-Pacific Forum on Science Learning and Teaching, 9(2), 1-22.

Cohen, L. y Marion, L. (1990). Métodos de investigación educativa. España: La Muralla.

Del Carmen, L. (2006). Los trabajos prácticos. Primer Taller de Actualización sobre los Programas de Estudio 2006. Buenos Aires: Secretaría de Educación Pública.

Gándara, M.; Gil, M. y Sanmartí, N. (2002). Del modelo científico de "adaptación biológica" al modelo de "adaptación biológica" en los libros de texto de enseñanza secundaria obligatoria. Revista Enseñanza de las Ciencias, 20(2), 303-314.

Gargallo, B.; Pérez, C.; Serra, B.; Sánchez, F. y Ros, I. (2007). Actitudes ante el aprendizaje y rendimiento académico en los estudiantes universitarios. Revista Iberoamericana de Educación, 1(42), 1-22.

Gil, D. y Valdés, C. (1996). La orientación de las prácticas de laboratorio como investigación: un ejemplo ilustrativo. Enseñanza de las Ciencias, 14(2), 155-163.

González, C.; García, S. y Martínez, C. (2003). ¿̇A qué contenidos relacionados con la fotosíntesis dan más importancia los textos escolares de secundaria? Enseñanza de las Ciencias, número extra, 77-88.

Herron (1971). The nature of scientific inquiry. School Review, 79, 171-212.

Jiménez, Llobera y Llitiós (2006). La atención a la diversidad en las prácticas de laboratorio de química: los niveles de abertura. Enseñanza de las Ciencias, 24 (1), $311-320$.

Köse, S. y Uşak, M. (2006). Determination of prospective science teachers' misconceptions: Photosynthesis and respiration in plants. International Journal of Environmental and Science Education, 1 (1), 25-52.

Leymonié, S. (2009). Aportes para la enseñanza de las Ciencias Naturales. Segundo Estudio Regional Comparativo y Explicativo. Unesco y Llece. Recuperado de http://www.oei.es/decada/180275s.pdf.

Manassero, M. y Vásquez, A. (2001). Instrumentos y métodos para la evaluación de las actitudes relacionadas con la ciencia, la tecnología y la sociedad. Enseñanza de las Ciencias, 1 (20), 15-27.

Maris, A. (2007). Cómo desean trabajar los alumnos en el laboratorio de biología. Un acercamiento a las propuestas didácticas actuales. Revista Iberoamericana de Educación, 42 (7), 1-13.

Pozo, J. y Gómez, M. (1994). La solución de problemas. Madrid: Aula xxı Santillana.

De Pro, B. (1999). La utilización de un modelo de planificación de unidades didácticas: el estudio de las disoluciones en la educación secundaria. Enseñanza de las Ciencias, 15(1), 35-50. 
Sánchez, G. y Valcárcel, M. (1993). Diseño de unidades didácticas en el área de ciencias experimentales. Enseñanza de las Ciencias, 17 (1), 33-44.

Sanmartí, N. (2002). Didáctica de las ciencias en la educación secundaria obligatoria. Madrid: Síntesis.

Stake, R. (1998). Investigación con estudio de casos. Madrid: Morata.

Toulmin, S. (1977). Comprensión humana. Madrid: Alianza Universidad.

Vázquez, A. y Manassero, M. (1997). Análisis empírico de dos escalas de motivación escolar. Revista Electrónica de Motivación y Emoción, 3(5), 1-38.

Wandersee, J. (1986). Can the history of science help science educators anticipate students' misconceptions? Journal of Research in Science Teaching, 23(7), 581-597. 